\title{
Study on superabsorbent composites XVII. Preparation and characterization of poly(acrylic acid)/attapulgite/Na- montmorillonite superabsorbent composites
}

\author{
Junping Zhang, ${ }^{12}$ Kun Zhang, ${ }^{1}$ Aiqin Wang $^{1 *}$ \\ ${ }^{1 *}$ Center for Eco-material and Green Chemistry, Lanzhou Institute of Chemical \\ Physics, Chinese Academy of Sciences, Lanzhou, 730000, P.R. China; tel.: +86-931- \\ 4968118; fax: +86-931-8277088; e-mail: aqwang@lzb.ac.cn \\ ${ }^{2}$ Graduate University of the Chinese Academy of Sciences, Beijing, 100049, P.R. \\ China
}

(Received: 3 January, 2008; published: 9 October, 2008)

\begin{abstract}
A novel superabsorbent composite simultaneously incorporated with both attapulgite and $\mathrm{Na}$-montmorillonite was prepared by aqueous polymerization using $N, N^{\prime}$-methylenebisacrylamide as a crosslinker and ammonium persulfate as an initiator. The effects of the clay content on swelling ability of the superabsorbent composite were investigated. The superabsorbent composite were characterized by IR, XRD and SEM. The results indicate that $\mathrm{Na}$-montmorillonite was exfoliated in the composite, whereas, attapulgite kept its crystalline structure. The content of clay has great influence on surface morphology and water absorbency of the superabsorbent composite, and the collaborative effect of attapulgite and $\mathrm{Na}$ montmorillonite on swelling ability is superior to that of single one.
\end{abstract}

\section{Introduction}

Superabsorbents are crosslinked network of hydrophilic polymers that can absorb and retain a lot of aqueous fluids, and the absorbed water is hardly removable even under some pressure. Superabsorbents are widely used in many fields, such as hygienic products, horticulture, gel actuators, drug-delivery systems and coal dewatering [1-5] due to excellent properties when compared to traditional water absorbing materials (such as sponge, cotton and pulp, etc.).

Recently, clay has become the focus for the preparation of superabsorbent composite in order to improve swelling properties, enhance gel strength and reduce production cost of corresponding superabsorbents. Clays, including montmorillonite [6], kaolin [7], mica [8, 9], attapulgite [10] and sericite [11], have already been incorporated into superabsorbent polymeric network and encouraging results have been observed. However, all the studies reported until now only introduced one kind of clay into the polymeric network to the best of our knowledge. As has been reported by Lin et al., introducing both montmorillonite and $\mathrm{SiO}_{2}$ simultaneously into polyacrylonitrile could further strengthen the material property compared with those incorporated with only montmorillonite or $\mathrm{SiO}_{2}$ [12].

Montmorillonite has been widely used for the preparation of nanocomposites. Attapulgite, a kind of hydrated octahedral layered magnesium aluminum silicate absorbent mineral, has exchangeable cations and reactive $-\mathrm{OH}$ groups on its surface 
[13]. It has been reported by Neaman et al. that rheological parameters of mixed palygorskite-montmorillonite suspensions changed regularly with changing their ratio [14]. Chen et al. had already proved that the silicate layers of montmorillonite could interact with nano-fibres of attapulgite [15] and form clay-clay nanocomposites.

Considering the interaction between montmorillonite and attapulgite may influence swelling ability of prepared superabsorbent composites, the collaborative effects of them were investigated by introducing attapulgite and $\mathrm{Na}$-montmorillonite into the poly(acrylic acid) system simultaneously on the basis of our previous work about superabsorbent composite [16-21].

\section{Results and discussion}

\section{IR spectra}

IR spectra of APT, Na-MMT and the corresponding superabsorbent composites incorporated with various amount of APT are shown in Figure 1 and Figure 2, respectively. The absorption bands in the range of $3400 \mathrm{~cm}^{-1}-3700 \mathrm{~cm}^{-1}$ are attributed to the stretching of $-\mathrm{OH}$ as shown in Figure 1. The absorption bands at $3417 \mathrm{~cm}^{-1}$ and $1643 \mathrm{~cm}^{-1}$ attributed to $-\mathrm{OH}$ stretching and bending of $\mathrm{H}_{2} \mathrm{O}$, respectively, which indicates the existence of $\mathrm{H}_{2} \mathrm{O}$ in their structure. The absorption bands at $1030 \mathrm{~cm}^{-1}$ and $1039 \mathrm{~cm}^{-1}$ for APT and Na-MMT are attributed to the Si-O stretching. The absorption bands in Figure 1 in the range of $970 \mathrm{~cm}^{-1}-450 \mathrm{~cm}^{-1}$ are attributed to $-\mathrm{OH}$ bending and $\mathrm{M}-\mathrm{O}$ ( $\mathrm{M}$ denotes $\mathrm{Si}$ or other metal cations exists in these two clays). The partial neutralization of PAA is confirmed in Figure 2 (e) by the appearance of absorption bands at $1574 \mathrm{~cm}^{-1}$ and $1409 \mathrm{~cm}^{-1}$ attributed to asymmetrical and symmetrical stretching of $-\mathrm{COO}^{-}$, respectively. After incorporation into the PAA polymeric network, all the bands of APT and Na-MMT were weakened or even disappeared; this indicates the reaction between clays and PAA during the polymerization process. This phenomenon had been observed according to $\mathrm{Li}$ et al. [16].

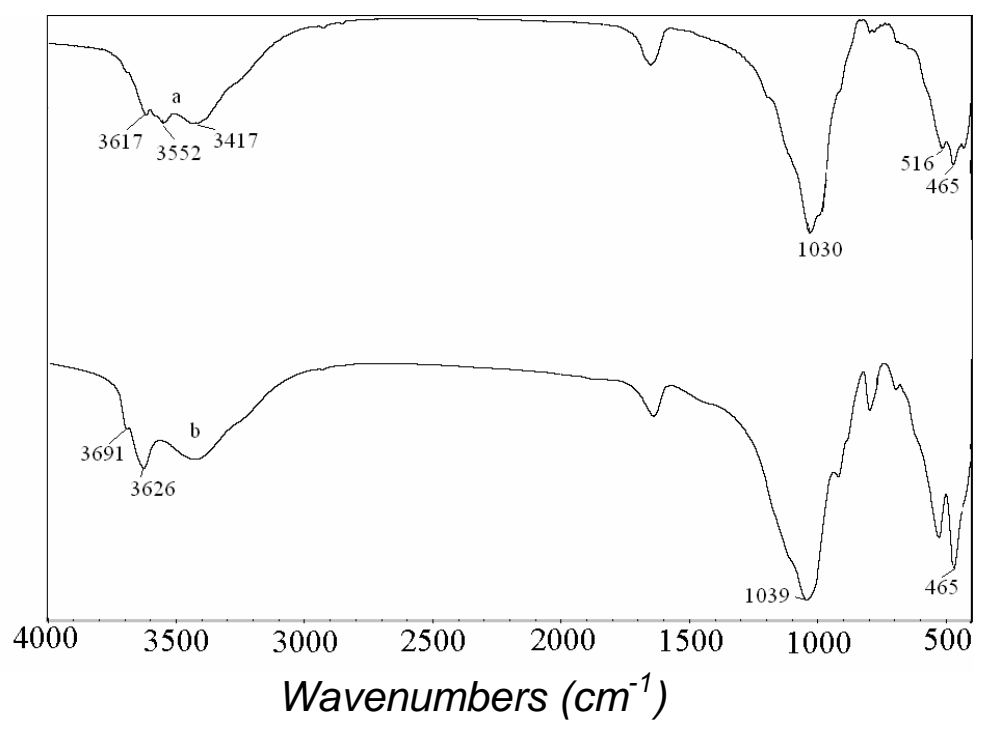

Fig. 1. IR spectra of the (a) APT and (b) Na-MMT.

It also can be seen from Figure 2 that intensity of the absorption bands of APT at $3617 \mathrm{~cm}^{-1}, 1035 \mathrm{~cm}^{-1}$ and $470 \mathrm{~cm}^{-1}$ increase with increasing APT content. In addition, 
asymmetrical stretching of $-\mathrm{COO}$ shifts from $1608 \mathrm{~cm}^{-1}$ to $1574 \mathrm{~cm}^{-1}$ with increasing APT content from 0 wt.\% to $30 \mathrm{wt} . \%$, which indicates that the introduced APT may have some influence on chemical environment of the hydrophilic - $\mathrm{COO}^{-}$group, and then on swelling ability of the superabsorbent composite.

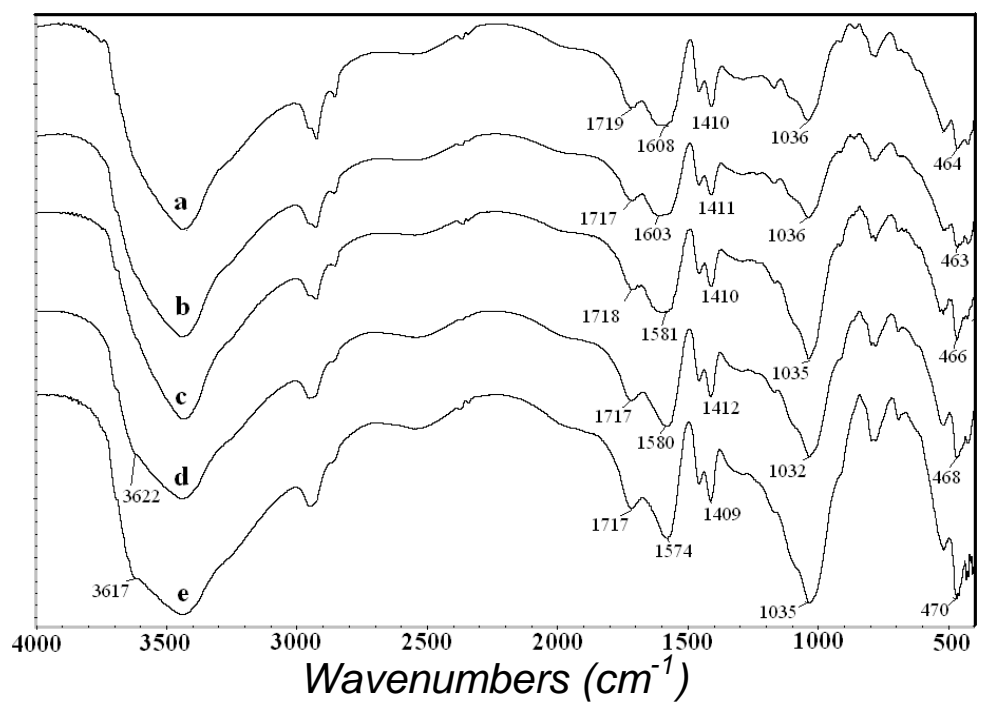

Fig. 2. IR spectra of the PAA/APT/Na-MMT superabsorbent composites incorporated with (a) 0 wt.\% APT, (b) 5 wt.\% APT, (c) 10 wt.\% APT, (d) 20 wt.\% APT, (e) 30 wt.\% APT. Na-MMT content in all the composites is $20 \mathrm{wt} . \%$

\section{$X R D$ analysis}

The interaction among APT, Na-MMT and PAA were also investigated by XRD. The XRD patterns of Na-MMT, APT and PAA/APT/Na-MMT superabsorbent composites incorporated with various amount of APT are presented in Figure 3.

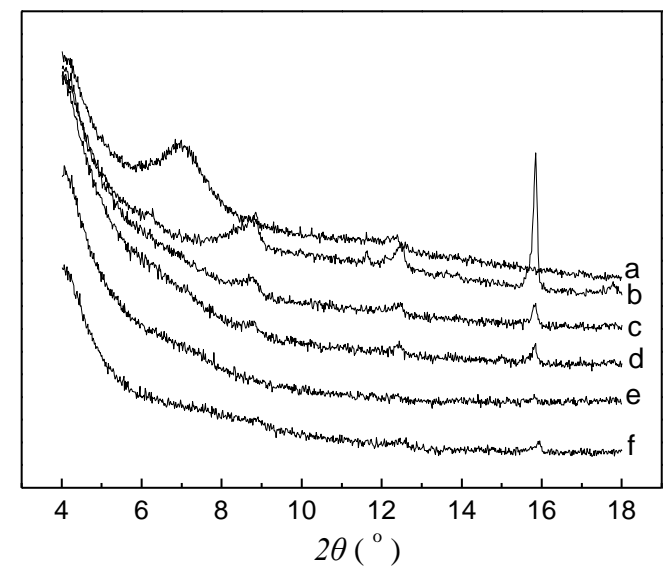

Fig. 3. XRD patterns of (a) Na-MMT, (b) APT and PAA/APT/Na-MMT superabsorbent composites incorporated with (c) 30 wt.\% APT, (d) 20 wt.\% APT, (e) 10 wt.\% APT and (f) 5 wt. $\%$ APT. Na-MMT content in all the composites is 20 wt. $\%$

The typical diffraction peaks of Na-MMT and APT are at 6.94 (basal spacing, 1.27 $\mathrm{nm}$ ) and $8.41^{\circ}$ (basal spacing, $1.05 \mathrm{~nm}$ ), respectively. As can be seen, the typical 
diffraction peak of Na-MMT disappeared no matter how much APT was incorporated. Slices of Na-MMT are in exfoliated state in aqueous medium, thus, nanofibres of APT and AA could exist among these exfoliated slices before polymerization. During the polymerization process, the slices of Na-MMT would like to contract to its original structure, however, the existence of APT and PAA restricts its contraction, and thus kept the exfoliated structure of Na-MMT. The typical diffraction peak of APT can not be seen clearly when its content is lower than $10 \mathrm{wt} . \%$. With increasing APT content to $20 \mathrm{wt} . \%$, the diffraction peak of APT can be seen clearly, which indicates that the interaction among APT, Na-MMT and AA as well as the polymerization have no obvious influence on crystalline structure of APT. This is attributed to the structure difference between Na-MMT and APT. The continuous octahedral sheets of APT are not convenient for the penetration of Na-MMT and polymer chains. This result is in conformity with our previous study on attapulgite based superabsorbent composites [17]. The result observed from the XRD patterns of clays and corresponding superabsorbent composites reveal that Na-MMT and APT play different role in the superabsorbent composites. Thus, they may have different influence on absorbing ability of the corresponding superabsorbent composites.

\section{Surface morphology}

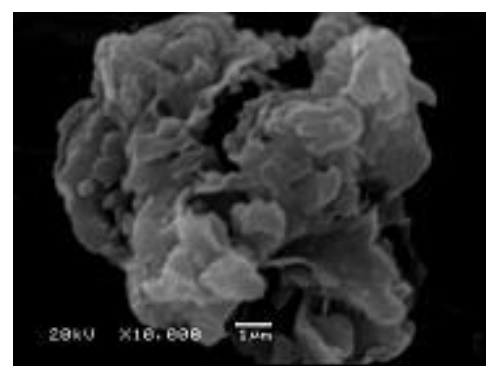

(a)

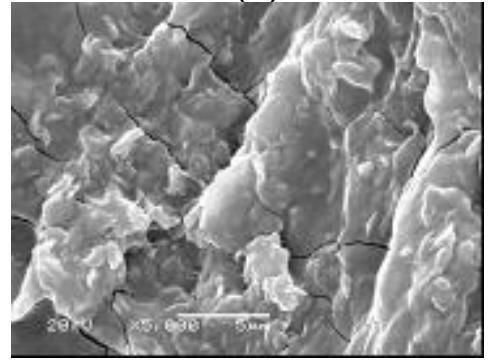

(d)

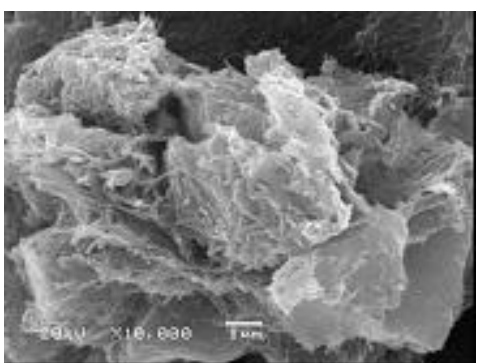

(b)

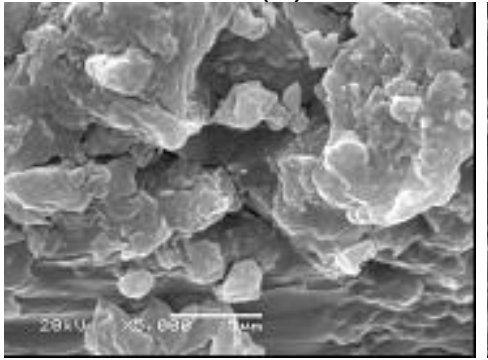

(e)

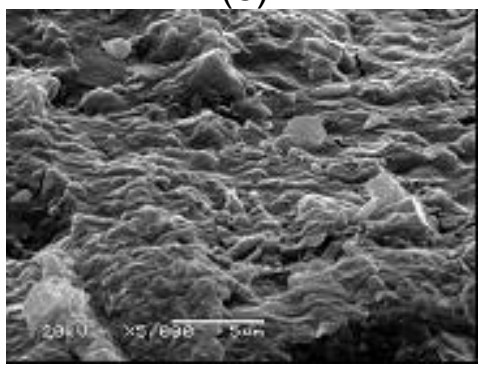

(g)

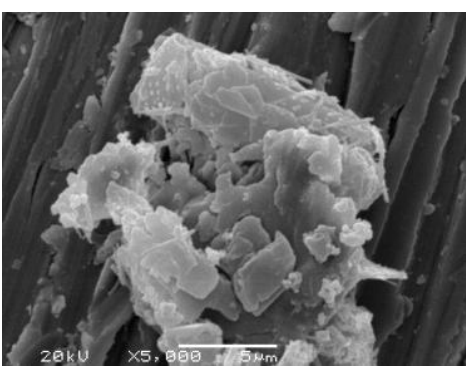

(c)

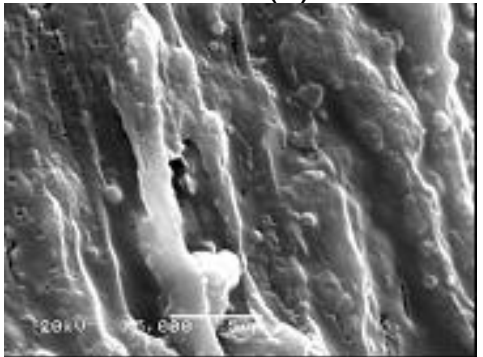

(f)

Fig. 4. SEM micrographs of (a) Na-MMT, (b) APT and PAA/APT/Na-MMT superabsorbent composites incorporated with (c) 0 wt.\% APT, (d) 5 wt.\% APT, (e) 10 wt.\% APT, (f) 20 wt.\% APT and (g) 30 wt.\% APT. Na-MMT content in all the composites is 20 wt. \% 
SEM micrographs of Na-MMT, APT and PAA/APT/Na-MMT superabsorbent composites incorporated with various amount of APT are shown in Figure 4. As can be seen, APT shows a fibrous surface and Na-MMT has a leprose surface. All the superabsorbent composites show accidented surface morphology. With increasing APT content from 0wt.\% to 30wt.\%, surface morphology of the superabsorbent composites changes from flaky (like slices of Na-MMT) to fluctuant and directional (like fibrous APT). With the increase of APT content, the percentage of Na-MMT in the composite decreases and the surface morphology changes is attributed to both their content. It can be concluded from Figure 4 that the content of APT and Na-MMT has great influence on surface morphology of the superabsorbent composites. These surface morphology changes on introducing various amount of APT and Na-MMT may have some influence on swelling ability of corresponding superabsorbent composites.

\section{Effects of Na-MMT and APT content on water absorbency}

Figure 5 shows variation of water absorbency for the PAA/APT/Na-MMT superabsorbent composite with Na-MMT content at a fixed APT content. As can be seen, the water absorbency increases with increasing Na-MMT from 0 wt.\% to 5 wt.\%. The $\mathrm{Na}^{+}$cations between layers of $\mathrm{Na}-\mathrm{MMT}$ could ionize when the PAA/APT/Na-MMT superabsorbent composite is immersed in water, which enhances hydrophilicity of the superabsorbent composite and makes it swell more [22]. The water absorbency decreases evidently from $918 \mathrm{~g} \mathrm{~g}^{-1}$ to $550 \mathrm{~g} \mathrm{~g}^{-1}$ with further increasing of Na-MMT content from $5 \mathrm{wt} . \%$ to $30 \mathrm{wt} . \%$. It has been suggested by Wu et al. [23] that the Na-MMT particles could fill up the polymer network or be chemically bound to organic polymer, which increases crosslink density of the superabsorbent composite and forms a tighter network, thereby decreasing the water absorbency.

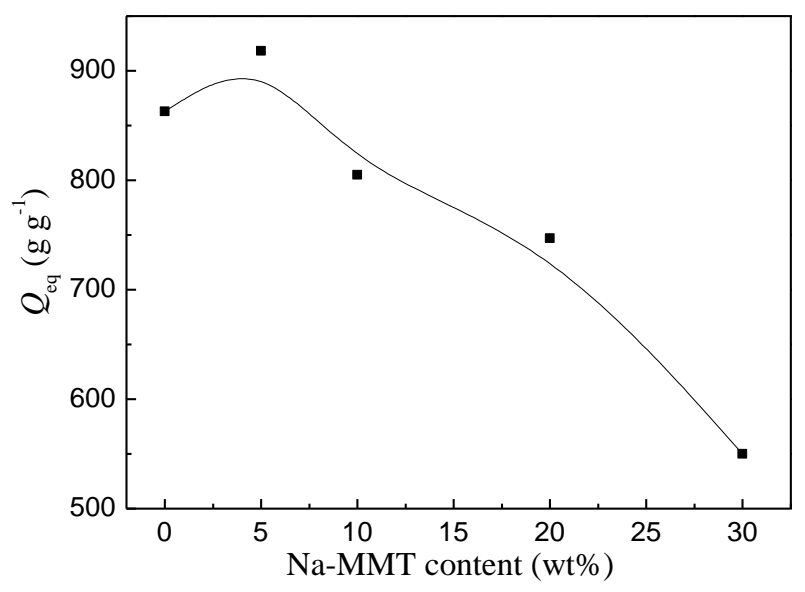

Fig. 5. Variation of water absorbency for the PAA/APT/Na-MMT superabsorbent composite with Na-MMT content. APT content in all the composites is $20 \mathrm{wt} . \%$

At a fixed Na-MMT content, water absorbency for the superabsorbent composite increases from $682 \mathrm{~g} \mathrm{~g}^{-1}$ to $805 \mathrm{~g} \mathrm{~g}^{-1}$ with increasing APT content from 0 wt. $\%$ to 10 wt.\%, and then decreases to $649 \mathrm{~g} \mathrm{~g}^{-1}$ with further increase of APT content to 30 wt.\% as shown in Figure 6. This trend of water absorbency with increasing APT 
content is in conformity with our previous study [16]. Comparing Figure 5 with Figure 6 , it can be seen that the composite incorporated with a higher APT content has higher water absorbency when the total amount of APT and Na-MMT is the same. For example, the composite incorporated with $20 \mathrm{wt} \%$ APT and $5 \mathrm{wt} . \%$ Na-MMT has a water absorbency of $918 \mathrm{~g} \mathrm{~g}^{-1}$, whereas water absorbency for the composite incorporated with $5 \mathrm{wt} . \%$ APT and $20 \mathrm{wt} \% \mathrm{Na}$-MMT is only of $715 \mathrm{~g} \mathrm{~g}^{-1}$.

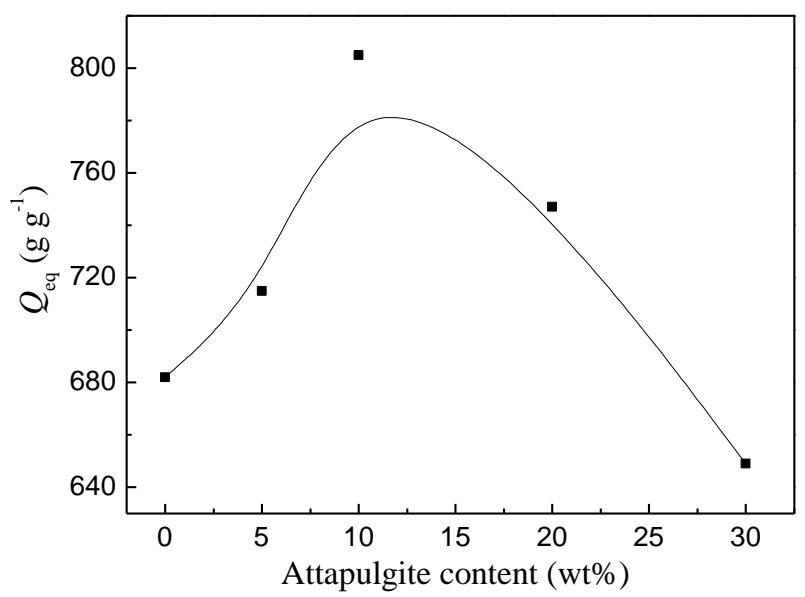

Fig. 6. Variation of water absorbency for the PAA/APT/Na-MMT superabsorbent composite with APT content. Na-MMT content in all the composites is $20 \mathrm{wt} . \%$

It can be deduced from Figures 5 and 6 that APT and Na-MMT have different influence on swelling ability of the superabsorbent composite. This may be attributed to their crystalline structure difference and the corresponding different roles they play in the superabsorbent composite. APT and PAA chains could intercalate into layers of Na-MMT and cause its exfoliation, which generates a lot of crosslink points in the network. However, the interaction among APT, Na-MMT and PAA just occurs on the surface of APT owing to its continuous octahedral structure, which restricts the penetration of Na-MMT and PAA chains, and then relatively less amount of crosslink points are formed. It also can be concluded from Figures 5 and 6 that the APT-NaMMT synergy is superior to the single one. The superabsorbent composite incorporated with 20 wt.\% APT and 5 wt.\% Na-MMT acquires the highest water absorbency of $918 \mathrm{~g} \mathrm{~g}^{-1}$.

\section{Conclusions}

A novel superabsorbent simultaneously incorporated with two kinds of clays was synthesized among Na-MMT, APT and AA. APT and Na-MMT interacted with each other during the polymerization process. Na-MMT was exfoliated, whereas, APT kept its crystalline structure during the polymerization process. The amount of clay had great influence on surface morphology and swelling ability of the superabsorbent composite. APT and Na-MMT had different influence on swelling ability of the composite owing to their structure difference. The APT-Na-MMT synergy was found to be superior to the single one. 


\section{Experimental part}

\section{Materials}

Acrylic acid (AA, distilled under reduced pressure before use), ammonium persulfate (APS, recrystallized from distilled water before use) and $N, N^{\prime}$ methylenebisacrylamide (MBA, used as received) were supplied by Shanghai Reagent Corp. (Shanghai, China). Attapulgite (APT, supplied by Linze Colloidal Co., Gansu, China) was milled through a 320-mesh screen before use. Namontmorillonite (Na-MMT) with a cation-exchange capacity of $102.8 \mathrm{mmol} / 100 \mathrm{~g}$ was purchased from the Longfeng Montmorillonite Co. (Shandong, China) and was milled through a 320-mesh screen before use. Other agents used were all of analytical grade and all solutions were prepared with distilled water.

\section{Preparation of poly(acrylic acid)/attapulgite/Na-montmorillonite (PAA/APT/Na-MMT)} superabsorbent composites

A series of superabsorbent composites from AA, APT and Na-MMT micropowder were synthesized according to the following procedure. $7.20 \mathrm{~g} \mathrm{AA}$ with a fixed neutralization degree of $60 \%$ (neutralized at $0{ }^{\circ} \mathrm{C}$ with $4 \mathrm{M}$ sodium hydroxide solution) were introduced to $17.0 \mathrm{ml}$ distilled water in a $250 \mathrm{ml}$ four-neck flask, equipped with a stirring rod, a reflux condenser, a thermometer, and a nitrogen line. $16 \mathrm{mg}$ crosslinker (MBA) was added to the above monomer solution, and then appropriate amount of APT and Na-MMT were dispersed in the mixed solution. After being purged with nitrogen for $30 \mathrm{~min}$ to remove the oxygen dissolved from the solution, $80.2 \mathrm{mg}$ initiator (APS) was charged into the flask as the temperature was increased to $40^{\circ} \mathrm{C}$. The solution was stirred vigorously and gradually heated to $70{ }^{\circ} \mathrm{C}$ under nitrogen atmosphere for $3 \mathrm{~h}$ to complete the polymerization. After polymerization, the product was washed with excess distilled water for several times to remove any unreacted reactants. The depurative product was dried in an oven at $70{ }^{\circ} \mathrm{C}$ until weight of the product was constant. The products were milled and all samples used for test had a particle size in the range of 40-80 mesh.

\section{Measurement of water absorbency}

$0.05 \mathrm{~g}$ sample was immersed in excess distilled water $(500 \mathrm{ml})$ at room temperature for $8 \mathrm{~h}$ to reach swelling equilibrium. Swollen samples were then separated from unabsorbed water by filtering through a 100-mesh screen under gravity for $30 \mathrm{~min}$ and no blotting of samples occured. Water absorbency in distilled water of the superabsorbent composite, $Q_{\mathrm{eq}}$, was calculated using the following equation:

$$
Q_{\mathrm{eq}}=\frac{m_{2}-m_{1}}{m_{1}}
$$

where $m_{1}$ and $m_{2}$ are the weights of the dry sample and the swollen sample, respectively. $Q_{\mathrm{eq}}$ is calculated as grams of water per gram of sample.

\section{Characterization}

IR spectra of samples were taken as KBr pellets using a Thermo Nicolet NEXUS TM spectrophotometer. The micrographs of samples were taken using SEM (JSM5600LV, JEOL, Ltd.). Before SEM observation, all samples were fixed on aluminum stubs and coated with gold. Powder XRD analyses of the specimens were performed 
using an X-ray power diffractometer with $\mathrm{Cu}$ anode (PAN alytical Co. X'pert PRO), running at $40 \mathrm{kV}$ and $30 \mathrm{~mA}$, scanning from $4^{\circ}$ to $18^{\circ}$ at $3^{\circ} / \mathrm{min}$.

\section{Acknowledgements}

This work was financially supported by the West Light Foundation and the Western Action Project of CAS (No. KGCX2-YW-501) and "863" Project of the Ministry of Science and Technology, P. R. China (No. 2006AA03Z0454).

\section{References}

[1] Buchholz, F. L.; Graham, T. "Modern Superabsorbent Polymer Technology", Wiley-VCH, NewYork, 1998.

[2] Dorkoosh, F. A.; Brussee, J.; Verhoef, J. C.; Borchard, G.; Tehrani, M. R.; Junginger, H. E. Polymer 2000, 41, 8213.

[3] Shiga, T.; Hirose, Y.; Okada, A.; Kurauchi, T. J. Appl. Polym. Sci. 1992, 44, 249.

[4] Raju, K. M.; Raju, M. P.; Mohan, Y. M. Polym. Int. 2003, 52, 768.

[5] Ende, M.; Hariharan, D.; Peppas, N. A. React. Polym. 1995, 25, 127.

[6] Kabiri, K.; Zohuriaan-Mehr M. J. Macromol. Mater. Eng. 2004, 289, 653.

[7] Wu, J. H.; Wei, Y. L.; Lin, S. B. Polymer 2003, 44, 6513.

[8] Lin, J. M.; Wu, J. H.; Yang, Z.; Pu, M. L. Macromol. Rapid Commun. 2001, 22, 422.

[9] Lee, W. F.; Chen, Y. C. Eur. Polym. J. 2005, 41, 1605.

[10] Li, A.; Liu, R. F.; Wang, A. Q. J. Appl. Polym. Sci. 2005, 98, 1351.

[11] Wu, J. H.; Lin, J. M.; Zhou, M.; Wei, C. Macromol. Rapid Commun. 2000, 21, 1032.

[12] Yu, T. S.; Lin, J. P.; Xu, J. F.; Chen, T.; Lin, S. L. Polymer 2005, 46, 5695.

[13] Neaman, A.; Singer, A. Applied Clay Sci. 2004, 25, 121.

[14] Neaman, A.; Singer, A. Clays Clay Miner. 2000, 48, 713.

[15] Chen, T. H.; Xu, H. F.; Lu, A. H.; Peng, S. C.; Xu, X. C.; Wang, J. Q. Bull. Chinese Ceramic Soc. 2004, 1, 40.

[16] Li, A.; Wang, A. Q.; Chen, J. M. J. Appl. Polym. Sci. 2004, 92, 1596.

[17] Zhang, J. P.; Chen, H.; Wang, A. Q. Eur. Polym. J. 2005, 41, 2434.

[18] Zhang, J. P.; Li, A.; Wang, A. Q. Carbohyd. Polym. 2006, 65, 150.

[19] Zhang, J. P.; Wang, L.; Wang, A. Q. Macromol. Mater. Eng. 2006, 291, 612.

[20] Zheng, Y. A.; Wang, A. Q. e-Polymers 2007, no. 127.

[21] Zhang, J. P.; Wang, L.; Wang, A. Q. Ind. Eng. Chem. Res. 2007, 46, 2497.

[22] Li, W. F.; Yang, L. G. J. Appl. Polym. Sci. 2004, 92, 3422.

[23] Wu, J. H.; Lin, J. M.; Li, G. Q.; Wei, C. R. Polym. Int. 2001, 50, 1050. 